\title{
ESTUDO DE MOTOR UNIVERSAL COM NÚCLEOS OBTIDOS PELOS PROCESSOS DA METALURGIA DO PÓ PARA UTILIZAÇÃO EM FURADEIRAS MANUAIS
}

\author{
Roberto L.R. Da Cas $^{1}$, Moises de M. Dias ${ }^{2}$, Lirio Schaeffer. ${ }^{3}$, Philippe Pauletti ${ }^{4}$, Fernando M. Fernandes ${ }^{5}$, Carlos A. Ferreira ${ }^{6}$, \\ Sérgio D. Bittencourt. ${ }^{*}$ \\ ${ }_{1}^{1}$ Laboratório de Transformação Mecânica - LdTM, Universidade Federal do Rio Grande do Sul, 91501-970, Porto Alegre, Brasil. \\ 2 Instituto de Ciências Exatas e Tecnológicas - ICET, FEEVALE, 93352-000, Novo Hamburgo, Brasil. \\ ${ }_{3}^{3}$ Laboratório de Transformação Mecânica - LdTM, Universidade Federal do Rio Grande do Sul, 91501-970, Porto Alegre, Brasil. \\ ${ }^{4}$ Departamento de Engenharia Elétrica, Faculdade SATC, 88805-380, Criciúma, Brasil. \\ 5 Laboratório de Transformação Mecânica - LdTM, Universidade Federal do Rio Grande do Sul, 91501-970, Porto Alegre, Brasil. \\ 6 Departamento de Engenharia Elétrica, Faculdade SATC, 88805-380, Criciúma, Brasil. \\ 1 * Laboratório de Corrosão, Proteção e Reciclagem de Materiais - LACOR, Universidade Federal do Rio Grande do Sul, 91501-970, \\ Porto Alegre, Brasil. \\ E-mail*: sergio.bittencourt@ufrgs.br
}

Este trabalho teve por objetivo o estudo de ligas sinterizadas a partir de Fe puro, FeP, FeSi e FeNi obtidas a partir dos processos da Metalurgia do Pó para sua aplicação nos núcleos do rotor e estator de um motor universal, tendo como finalidade sua utilização em uma máquina furadeira manual. A pesquisa compreendeu a obtenção de curvas de histerese destas ligas, ensaios para avaliação de perdas elétricas e simulação em software de elementos finitos para obtenção de fluxo de entreferro e conjugado eletromagnético (torque). Os estudos teóricos foram realizados em um motor universal de topologia convencional e as simulações foram realizadas de forma a comparar este motor com os de núcleos fabricados a partir de chapas de aço laminadas.

Palavras-chave: Metalurgia do Pó; Motor Universal; Simulação por elementos finitos.

\section{Introdução}

As máquinas elétricas rotativas, na sua grande maioria, possuem os núcleos do rotor e do estator fabricados com chapas de aço baixo carbono laminadas e isoladas eletricamente [1].

Os métodos de acionamento destas máquinas tem apresentado grande evolução, devido ao desenvolvimento da eletrônica de potência e capacidade de processamento dos acionamentos eletrônicos, fazendo com que a partir de máquinas elétricas rotativas tradicionais se tenha desempenho equivalente a máquinas que utilizam novos materiais, como as de ímãs permanentes na sua concepção [1-3].

A pesquisa de novos materiais e processos de fabricação para substituir as chapas de aço baixo carbono laminadas busca melhores características, como eficiência na conversão de energia elétrica em mecânica, menores custos de fabricação pela simplificação do processo e maior aproveitamento de matéria prima.
O rotor e o estator construídos por metalurgia do pó são mais leves e com excelente acabamento superficial. Propriedades importantes de materiais magnéticos macios são baixa coercitividade, alta permeabilidade, alta indução de saturação e baixa perda energética por histerese [3].

Este trabalho tem como objetivo o estudo de um motor universal utilizando material maciço sinterizado nos núcleos do rotor e estator, comparando suas características e desempenho em relação aos convencionais núcleos de chapas laminadas por meio de simulações da máquina elétrica do motor universal, através do software de elementos finitos - FEMM versão 4.2 -. Serão comparadas com os núcleos tradicionais simulações com Fe puro e ligas com a mistura de Fe1\%P, Fe2\%P, Fe3\%P, Fe1\%Si, $\mathrm{Fe} 3 \% \mathrm{Si}, \mathrm{Fe} 5 \% \mathrm{Si}$ e $\mathrm{Fe}-50 \% \mathrm{Ni}$. 


\section{Motores Elétricos a partir da Metalurgia do Pó}

\subsection{Processos da Metalurgia do Pó e suas Variações}

No processo de fabricação por metalurgia do pó, incluindo núcleos do rotor e estator de máquinas elétricas rotativas, as peças são fabricadas a partir da mistura homogênea dos pós, previamente selecionados de acordo com as características (mecânicas, elétricas, magnéticas) desejadas para a aplicação requerida. Os processos básicos da metalurgia do pó são [4-5]:

1. Obtenção dos pós

2. Mistura

3. Compactação

4. Sinterização

5. Retificação (somente em alguns casos)

Após a mistura os pós são compactados em uma matriz, adquirindo a forma da cavidade desta, e, posteriormente são sinterizados em fornos com atmosfera controlada para adquirir consistência e resistência mecânica [4-5].

\subsection{Principais Propriedades Físicas Consideradas}

Podemos resumir em três as principais propriedades físicas para definição de determinado material e processo para a fabricação de núcleos de máquinas elétricas rotativas [3]:

1. Propriedades magnéticas;

2. Resistividade elétrica;

3. Propriedades mecânicas.

As propriedades que devem ser observadas são, principalmente, o comportamento da magnetização do material, a resistividade elétrica para determinação das perdas elétricas e as mecânicas, com o intuito de verificar se os núcleos suportarão os esforços mecânicos da máquina durante seu funcionamento e vida útil [3].

2.2.1 Propriedades Magnéticas dos Materiais Aplicados em Motores Elétricos

Tomando uma bobina com determinado material em seu núcleo e submetendo-a a circulação de uma corrente elétrica I, um campo magnético $\mathrm{H}$ será gerado no interior desta bobina e a peça irá sofrer uma indução magnética B [6].

Caso o material dos constituinte dos núcleos possuam características ferromagnéticas, muitas linhas de fluxo serão induzidas em seu interior, acarretando uma relação $\mathrm{BxH}$ típica em uma determinada curva. Esta é denominada de curva de histerese ou ciclo de histerese e é demonstrada na Figura 1. Estes materiais também são conhecidos como materiais magnéticos [69].

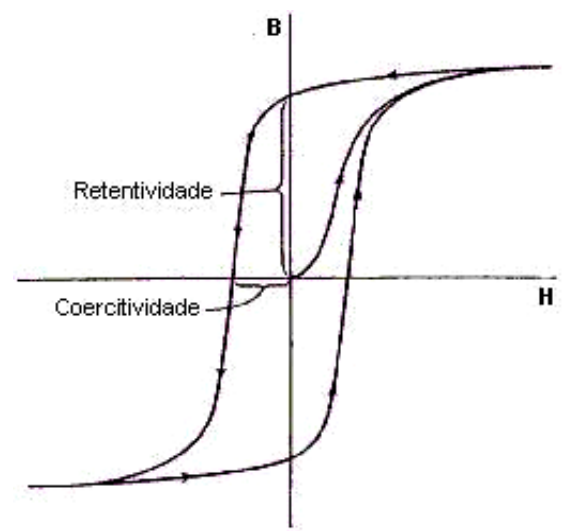

Figura 1 - Ciclo de histerese de um material magnético [9].

As propriedades magnéticas são obtidas a partir da curva de histerese, que relaciona campo magnético $\mathrm{H}$ aplicado a um material magnético, com a indução magnética $\mathrm{B}$ resultante. A Figura 2 relaciona os materiais magnéticos duros ou ímãs permanentes com os materiais magnéticos macios, como os aços magnéticos, que possuem um ciclo de histerese mais estreito com baixa coercitividade [7-9].

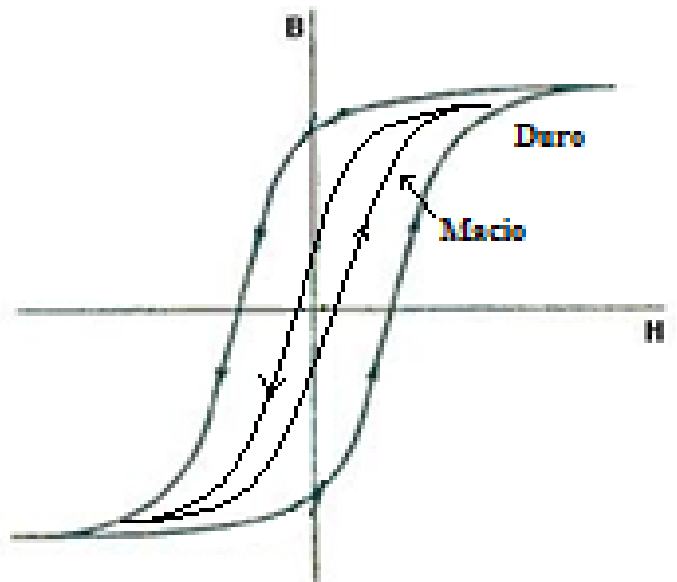

Figura 2 - Comparação entre material magnético macio e duro [8].

Conforme pode ser verificado na Figura 3, a curva do ciclo de histerese apresenta dois pontos de interesse para materiais aplicados em máquinas elétricas rotativas: o ponto $\mathrm{B}_{\mathrm{r}}$, denominado de indução magnética remanente ou retentividade, $\mathrm{o}$ qual representa a indução magnética residual sem campo magnético aplicado $(\mathrm{H}=0)$ e o ponto $\mathrm{H}_{\mathrm{c}}$, denominado de força coercitiva, que corresponde ao valor necessário de campo magnético para desmagnetizar o material. No SI a indução magnética $\mathrm{B}$ possui unidade em $\mathrm{Wb}$, enquanto campo magnético $\mathrm{H}$ é determinado em Tesla [7-9]. 


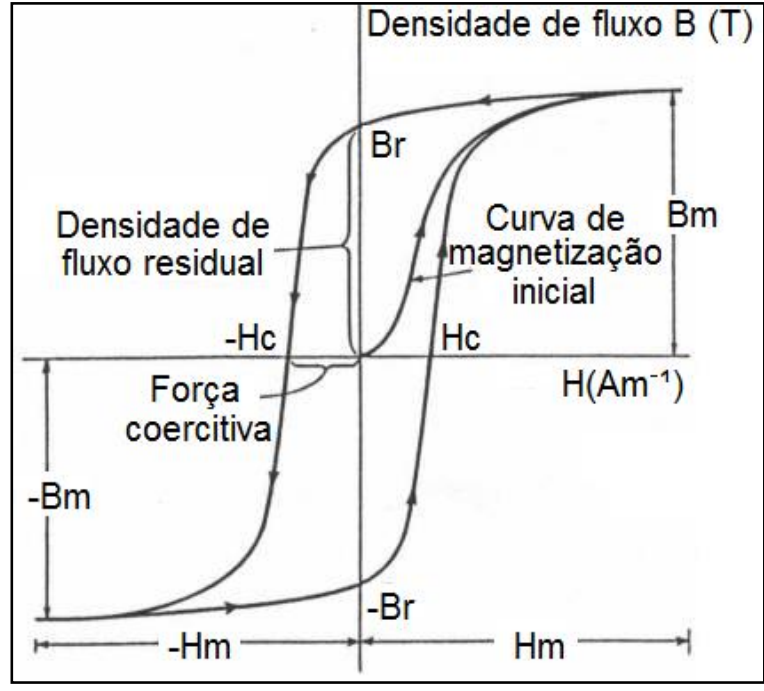

Figura 3 - Retentividade (Br) e coercitividade (Hc) [8].

\subsubsection{Resistividade Elétrica}

A resistividade elétrica é a capacidade que um determinado material tem de se opor à passagem de um fluxo de corrente elétrica. Em núcleos de máquinas elétricas busca-se alta resistividade para minimizar o efeito das correntes induzidas (correntes parasitas ou de Foucault) que levam ao aquecimento e à respectiva perda de rendimento [8].

Tradicionalmente, tanto o estator como o rotor são construídos com chapas laminadas e isoladas, uma vez que esta isolação entre chapas restringe as correntes induzidas a uma menor área de circulação. As perdas por correntes parasitas em um núcleo maciço são consideravelmente maiores que as perdas em núcleos obtidos a partir de chapas isoladas eletricamente. Quanto menor a espessura das chapas, menores são as correntes parasitas e menor é a perda de potência nestes núcleos. A redução das correntes induzidas, também, pode ser obtida a partir do aumento da resistência elétrica da peça, ou a partir do aumento da resistividade elétrica do material, uma vez que, resistência ou resistividade e corrente elétrica, são grandezas físicas inversamente proporcionais. Por este motivo, motores elétricos de alto rendimento são construídos com chapas de aço silício, que possui resistividade elétrica maior que o aço baixo carbono [10-11].

\subsubsection{Propriedades Mecânicas}

As principais grandezas a serem consideradas são dureza e ductilidade, com o intuito que os materiais utilizados para a fabricação dos núcleos de máquinas elétricas suportem os esforços solicitados pela carga e vibrações. Deste modo, devem ser realizados ensaios de dureza, ductilidade, curva de compressão x deformação ou curva de escoamento e avaliação da microestrutura $[3,8]$.

Em resumo, o material utilizado na construção dos núcleos do estator e do rotor devem apresentar as seguintes propriedades $[3,8]$ :

- Elevada Permeabilidade Magnética Relativa;

- Baixa Coercitividade Magnética;

- Elevada Resistividade Elétrica;

- Elevada Indução de Saturação;

- Dureza e ductilidade compatíveis com as vibrações as quais o motor é submetido.

\section{Motor Universal}

As principais partes de um motor universal são [12]:

- Armadura ou Rotor: em um motor, a armadura recebe a corrente proveniente de uma fonte elétrica externa fazendo a armadura girar. Por isso, esta parte do motor também é conhecida como rotor.

- Comutador: são responsáveis por alternar a corrente que passa pela armadura. O comutador é constituído por placas de cobre curvadas e fixadas, isoladamente, no eixo da armadura.

- Estator: a função do enrolamento de campo dentro do motor tem o objetivo de produzir a força magneto-motriz necessária para a geração do fluxo magnético necessário.

-Escovas: são conectores de grafite fixos, montados sobre molas para permitir que deslizem (escovem) sobre o comutador.

O estator é composto de uma estrutura ferromagnética com polos salientes aos quais são enroladas as bobinas que formam o campo, ou, por um ímã permanente. A Figura 4 mostra um motor universal parcialmente desmontado [12-13].

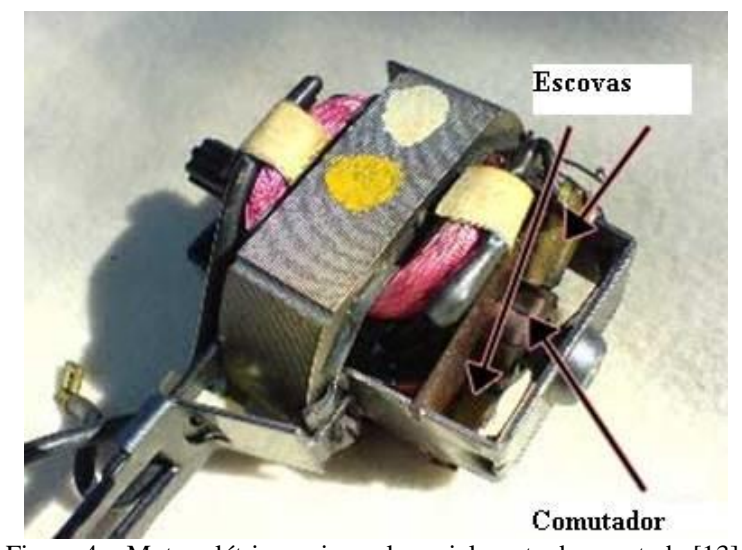

Figura 4 - Motor elétrico universal parcialmente desmontado [13] 
O rotor é um eletroímã constituído de um núcleo de ferro com enrolamentos em sua superfície que são alimentados por um sistema mecânico de comutação. Esse sistema é formado por um comutador, solidário ao eixo do rotor, que possui uma superfície cilíndrica com diversas lâminas às quais são conectados os enrolamentos do rotor; e por escovas fixas, que exercem pressão sobre o comutador e que são ligadas aos terminais de alimentação. O propósito do comutador é o de inverter a corrente na fase de rotação apropriada de forma a que o conjugado desenvolvido seja sempre na mesma direção [12-13].

Os enrolamentos do rotor compreendem bobinas de $\mathbf{n}$ espiras. Os dois lados de cada enrolamento são inseridos em sulcos com espaçamento igual ao da distância entre dois polos do estator, de modo que quando os condutores de um lado estão sob o polo norte, os condutores do outro devem estar sob o polo sul. As bobinas são conectadas em série através das lâminas do comutador, com o fim da última conectada ao início da primeira, de modo que o enrolamento não tenha um ponto específico [1213].

\subsection{Princípio de Funcionamento}

A Figura 5 mostra de maneira simplificada o funcionamento do motor universal. Quando alimentado por tensão alternada senoidal monofásica as correntes de campo e de armadura são as mesmas (enrolamentos estão em série) e quando uma muda sua polaridade, a outra muda ao mesmo tempo. Ou seja, o sentido do fluxo produzido pelo campo e o sentido da corrente de armadura mudam ao mesmo tempo, mantendo o sentido da força eletromagnética e, portanto, do torque [14].

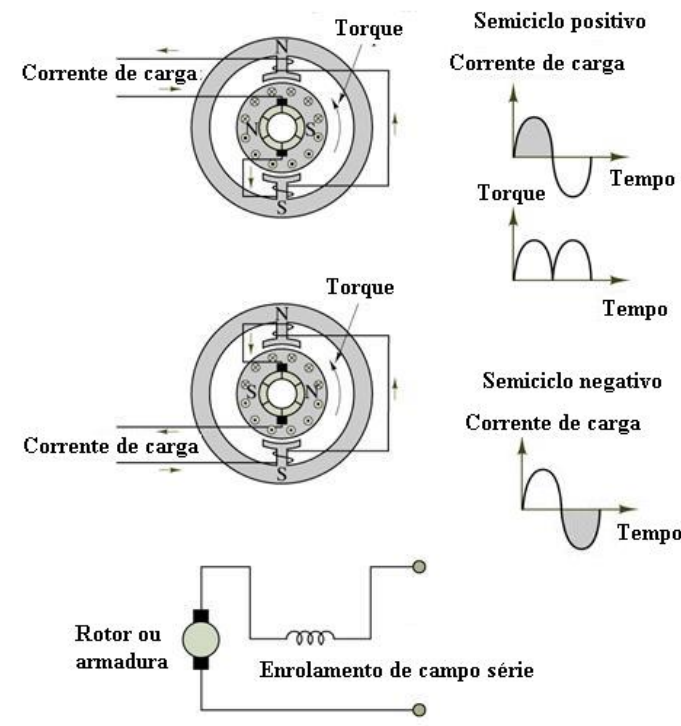

Figura 5 - Esquemático de funcionamento de um motor universal [14].
A função do comutador é trocar periodicamente (duas vezes a cada volta) o sentido da corrente na armadura de tal modo a garantir que o torque tenha sempre o mesmo sentido (horário, por exemplo) e impeça que a armadura fique parada em uma posição de equilíbrio. Sem o mecanismo da comutação, a espira da armadura iria estacionar na posição vertical, que é uma posição de equilíbrio. Quando a espira passa por uma posição de equilíbrio, o comutador muda a corrente, modificando, também, o sentido do torque, evitando que a espira volte para a posição de equilíbrio [14].

Os motores universais possuem características de desempenho específicas, o que determina o tipo de aplicação em que é usado. Essas características estão mostradas na Figura 6, em que se apresentam as curvas de torque e de velocidade em função da corrente de armadura [14].

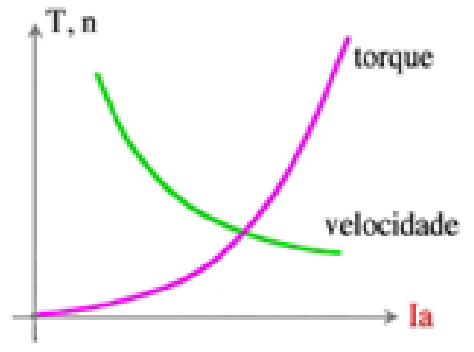

Figura 6 - Torque x velocidade em função da corrente.

Podemos observar que os motores universais possuem elevado torque em baixa rotação, para um determinado valor de corrente de armadura. Essa característica torna os motores universais adequados para acionamento, em corrente alternada, de vários eletrodomésticos (aspiradores de pó, liquidificadores, furadeiras), bem como, para acionamento de veículos elétricos de transporte de massa (trens, carros elétricos, metrôs) [14].

\section{Materiais e Métodos}

\subsection{Projeto do Motor}

O motor universal utilizado como referência foi o de uma furadeira padrão de mercado, de fabricação nacional, modelo KR505 do fabricante Black\&Decker (Figuras 7 e 8). Este tipo de máquina elétrica possui o rotor bobinado alimentado por um conjunto de escovas e estator bobinado. 

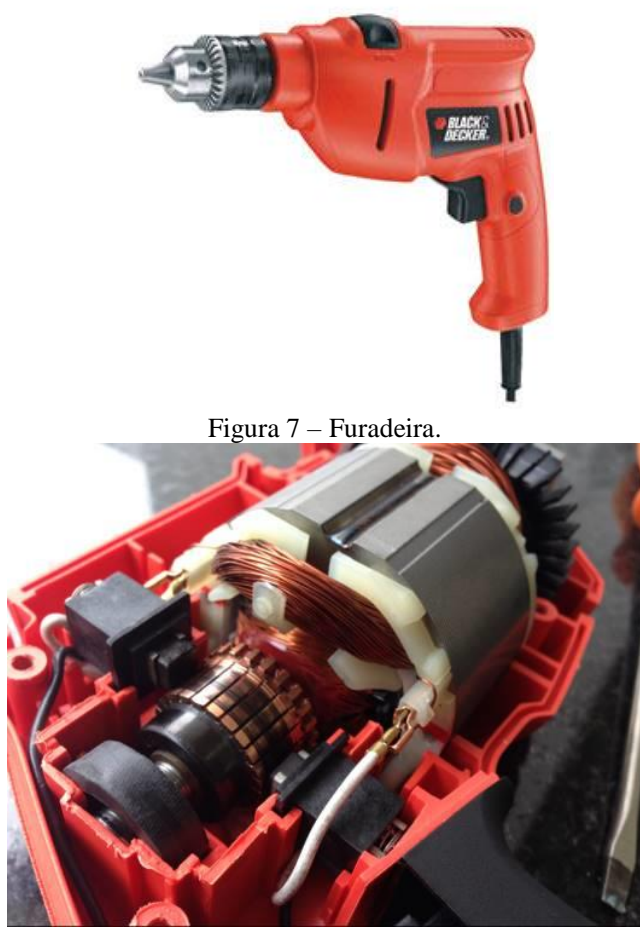

Figura 8 - Motor universal montado.

Principais características técnicas da furadeira:

- Voltagem: 220V

- Potência de entrada de energia: $480 \mathrm{~W}$

- Velocidade sem carga: 2800 rpm

- Tamanho do mandril: $3 / 8$ " (10mm)

As Figuras 9 e 10 apresentam o rotor e o estator fabricados com chapas laminadas com as bobinas.

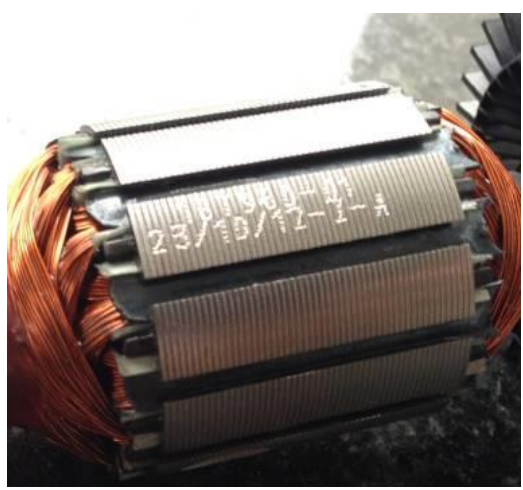

Figura 9 - Rotor com chapas laminadas.

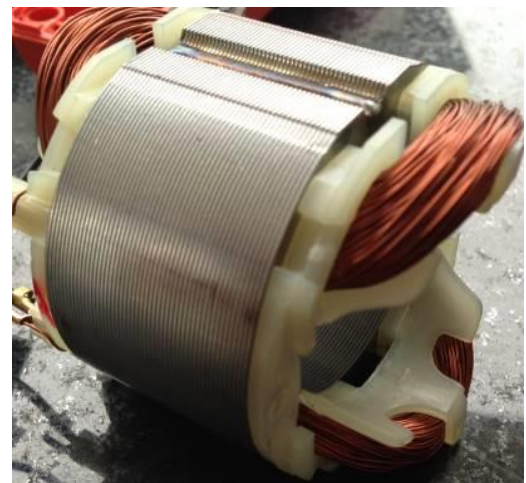

Figura 10 - Estator de chapas laminadas.

As Figuras 11 e 12 exibem os núcleos construídos pelos processos da Metalurgia do Pó.

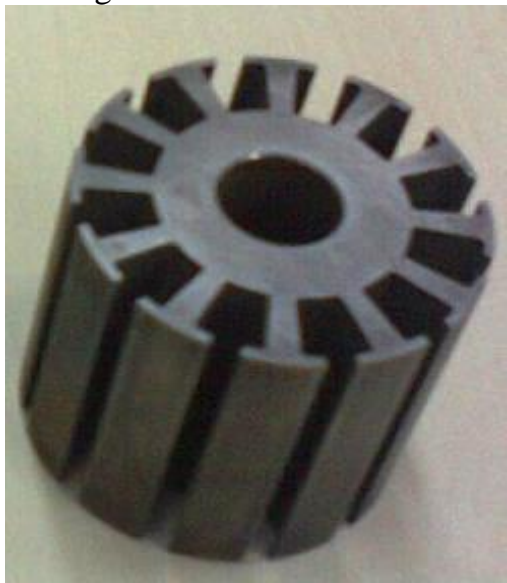

Figura 11 - Rotor de Fe sinterizado.

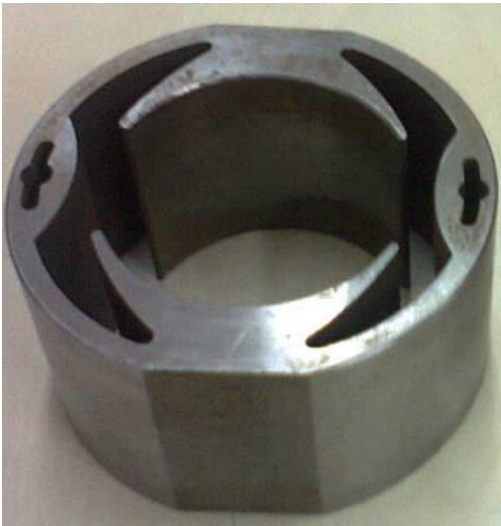

Figura 12 - Estator de Fe sinterizado.

Para a contagem do número de espiras e extração das medidas foram retiradas as bobinas e separados os núcleos do rotor e eixo, sendo que o eixo será inserido no rotor sinterizado, conforme elucidado na Figura 13. 


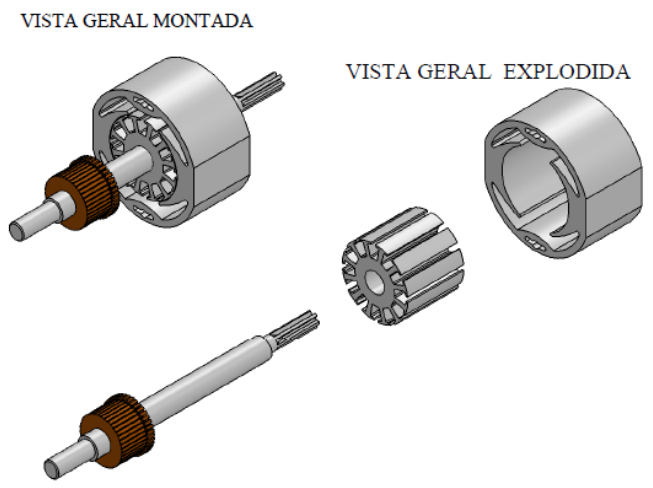

Figura 13 - Núcleos do estator, rotor e eixo do motor universal da furadeira.

As Figuras 14 e 15 exibem o projeto com as dimensões do núcleo do rotor para o motor universal da furadeira.

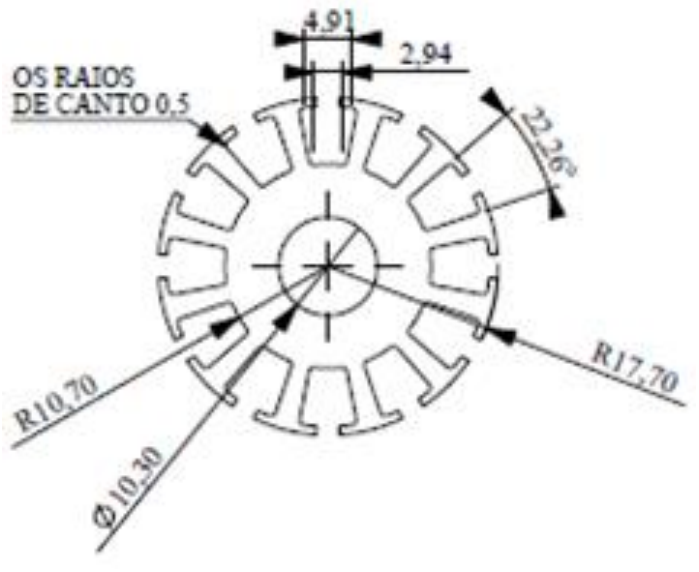

Figura 14 - Vista frontal com as dimensões do núcleo do rotor.

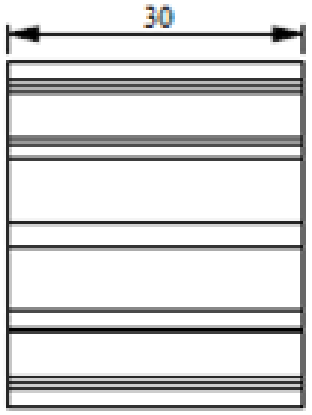

Figura 15 - Vista lateral com as dimensões do núcleo do rotor.

As Figuras 16 e 17 exibem o projeto com as dimensões do núcleo do estator para o motor universal da furadeira.

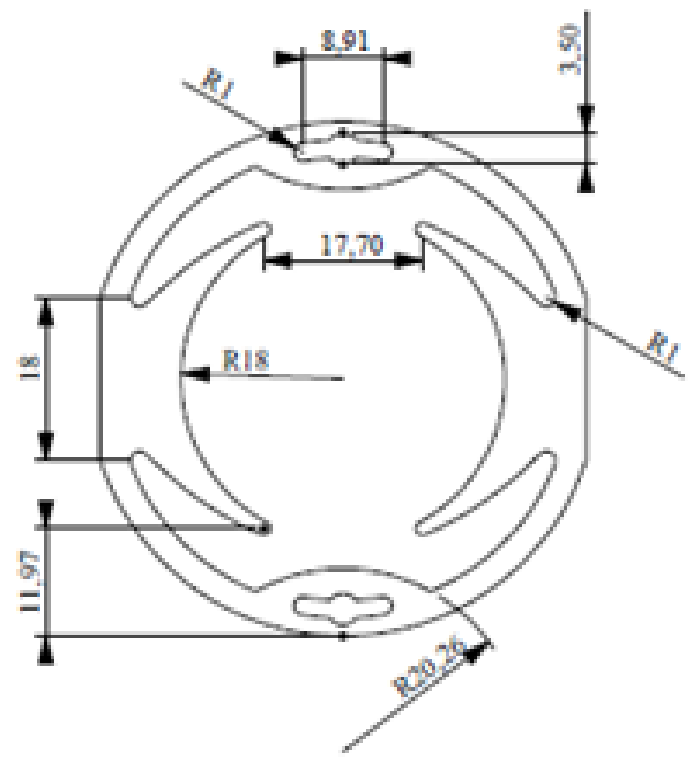

Figura 16 - Vista frontal com as dimensões do núcleo do estator.

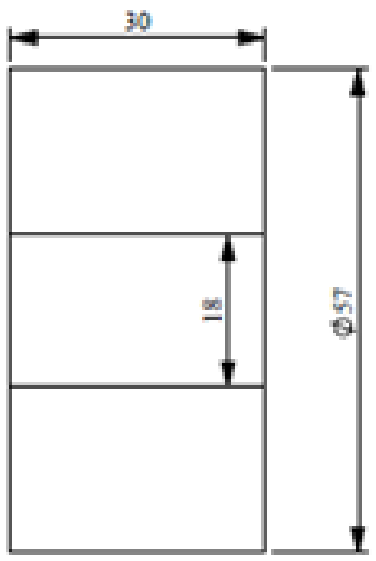

Figura 17 - Vista lateral com as dimensões do núcleo do estator.

\section{Resultados e Discussões}

\subsection{Simulações do Motor}

Para a simulação dos campos magnéticos nos núcleos do motor universal foi utilizado o software FEMM 4.2. A simulação do material magnético foi realizada a partir do projeto dimensional do motor, tendo como base corrente de 2,18 A, considerando-se um estator de dois polos e propriedades das bobinas do motor original:

-Rotor: Fio 30AWG 32 espiras, passe 6.

-Estator: Fio 26AGW 192 espiras. 
Nos gráficos das Figuras 18 e 19, respectivamente, são apresentados os resultados para as linhas de fluxo magnético nos núcleos do rotor e do estator do motor com material laminado e sinterizado.

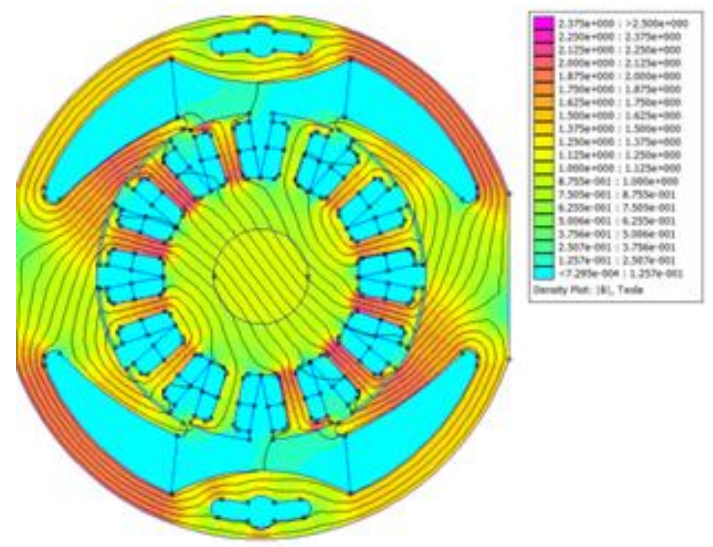

Figura 18 - Fluxo magnético no motor com material laminado.

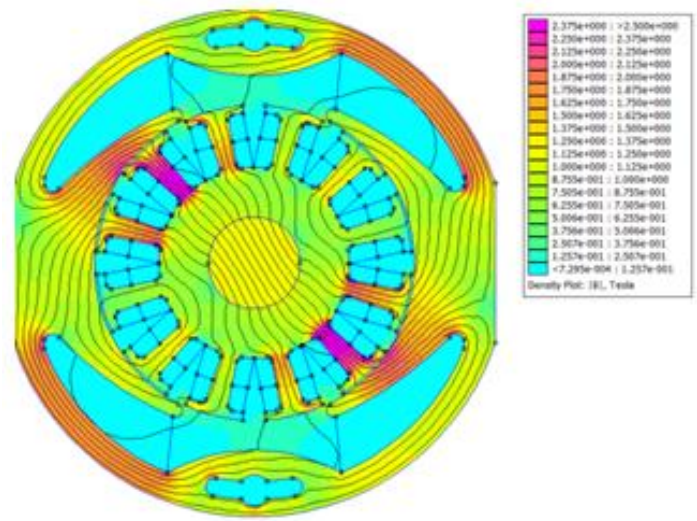

Figura 19 - Fluxo magnético no motor com liga sinterizada (Fe2\%P).

A Tabela 1 e as Figuras 20 e 21 apresentam o comparativo entre o torque e a densidade de fluxo máximos obtidos através das simulações realizadas no software por elementos finitos FEMM 4.2.

Tabela 1 - Comparativo entre torque e densidade de fluxo máximo.

\begin{tabular}{|c|c|c|}
\hline Núcleo & $\begin{array}{c}\text { Torque } \\
\text { (N.m) }\end{array}$ & $\begin{array}{c}\text { Fluxo Máximo } \\
\text { (T) }\end{array}$ \\
\hline $\begin{array}{c}\text { Chapas } \\
\text { M15-Steel }\end{array}$ & 0,379 & 2,25 \\
\hline Fe (puro) & 0,225 & 2,69 \\
\hline Fe1\%P & 0,276 & 3,07 \\
\hline
\end{tabular}

\begin{tabular}{|c|c|c|}
\hline Fe2\% $P$ & 0,247 & 3,69 \\
\hline $\mathbf{F e 3 \%} \mathbf{P}$ & 0,245 & 3,09 \\
\hline $\mathbf{F e 1 \%} \mathbf{S i}$ & 0,206 & 2,77 \\
\hline $\mathbf{F e 3 \%} \mathbf{S i}$ & 0,158 & 2,34 \\
\hline $\mathbf{F e 5 \%} \mathbf{S i}$ & 0,15 & 2,35 \\
\hline $\mathbf{F e 5 0 \%} \mathbf{N i}$ & 0,203 & 2,78 \\
\hline
\end{tabular}

\section{TORQUE (N.m)}

- TORQUE (N.m)

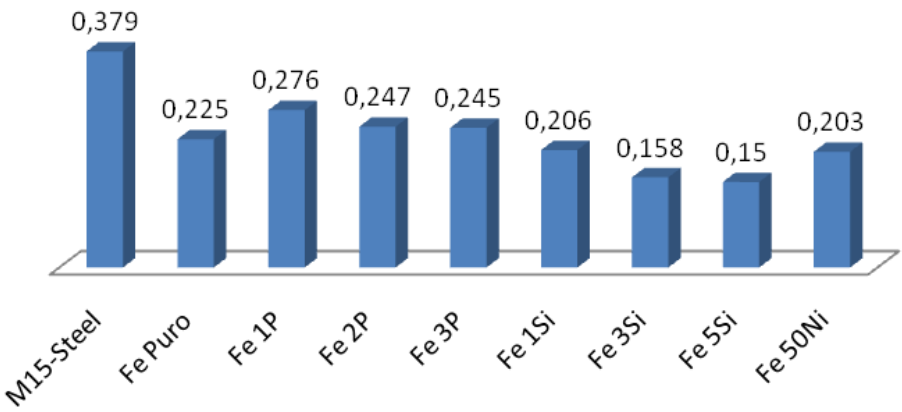

Figura 20 - Comparativo entre o torque das ligas estudadas.

\section{DENSIDADE DE FLUXO MÁXIMO (T)}

= DENSIDADE DE FLUXO MÁXIMO (T)

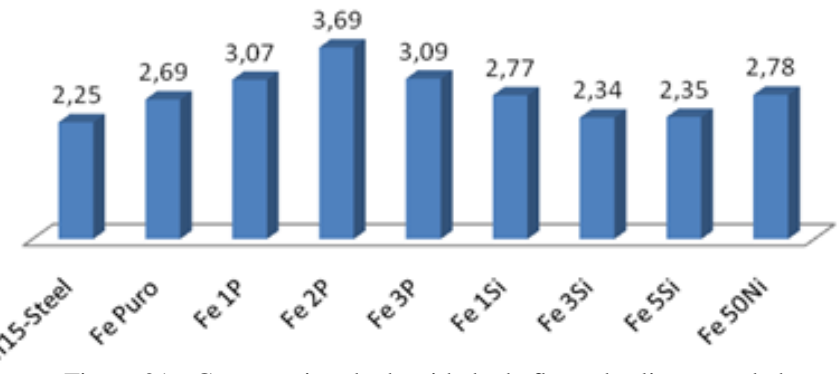

Figura 21 - Comparativo da densidade de fluxo das ligas estudadas.

\section{Conclusões}

Um material obtido por metalurgia do pó, para ser utilizado em núcleos de máquinas elétricas, em condições de substituir o tradicional pacote de chapas laminadas, deve possuir as seguintes características [15]:

a. Propriedades magnéticas dos sinterizados (permeabilidade e indução de saturação elevadas e baixa coercitividade).

b. Elevada resistividade elétrica (característica dos materiais compósitos). 
A partir dos resultados da simulação no FEMM 4.2, verifica-se que não existem zonas de saturação elevadas nos núcleos do motor, o que torna o projeto viável em termos de campos magnéticos.

A obtenção de peças magnéticas a partir do pó de ferro com uma adição de pequena quantidade de fósforo mostraram melhorias nas propriedades magnéticas analisadas.

As simulações realizadas com o software FEMM 4.2 mostraram que a fabricação de um motor universal com núcleos obtidos pelos processos da metalurgia do pó apresentam possibilidade técnica para sua construção utilizando-se Fe1\%P ou Fe2\%P, uma vez que as interações magnéticas entre o conjunto rotor-estator não apresentaram níveis elevados de saturação dos campos magnéticos.

Comparando os materiais obtidos por metalurgia do pó, observou-se que o composto $\mathrm{Fe} 2 \% \mathrm{P}$ apresentou melhor desempenho e propriedades magnéticas superiores em comparação aos demais compostos, sendo potencialmente viável para a aplicação na substituição aos atuais núcleos produzidos por chapas de aço.

\section{Agradecimentos}

Os autores agradecem a CAPES e ao CNPq pela bolsa e financiamento do projeto.

\section{STUDY OF UNIVERSAL MOTOR WITH CORES OBTAINED BY THE PROCESSES OF POWDER METALLURGY FOR USE IN MANUAL DRILLS.}

ABSTRACT: This work aimed to the study of sintered alloys from $\mathrm{Fe}$ pure, $\mathrm{FeP}, \mathrm{FeSi}$ and $\mathrm{FeNi}$ obtained from the processes of Powder Metallurgy for their application in cores of rotor and stator of a universal motor, with the purpose of its use in a manual drill machine. The research included obtaining hysteresis curves of these alloys, trials for evaluation of electrical losses and simulation in finite element software to obtain the air-gap flux and electromagnetic conjugate (torque). The theoretical studies were performed on a universal motor of conventional topology and the simulations were performed in order to compare this motor with the manufactured cores from rolled steel plates.

Keywords: Powder Metallurgy; Universal Motor; Finite Element Simulation.

\section{Referências}

[1] CHAPMAN, S. J. Electric Machinery Fundamentals. 4 ed. Mcgraw-Hill, 2003., $770 \mathrm{p}$.
[2]http://www.metalurgiadopo.com.br/Paginas/detalhes.asp?iType=1\&iPic=4, acessado em 26/10/2015

[3] BITTENCOURT, S. D., DIAS, M. M., SCHAEFFER, L. Ligas magnéticas sinterizadas usadas em máquinas elétricas rotativas, Novas Edições Acadêmicas, $2015,105 p$

[4] GERMAN, R.M. Powder Metallurgy Science. New Jersey, Metal Powder Industries Federation, 1984, 279p

[5] MORO, N.; AURAS, A. P. Metalurgia do Pó e o futuro da indústria Florianópolis: Centro Federal de Educação Tecnológica de Santa Catarina, Curso Técnico de Mecânica Industrial, 2007. 28 p.

[6] BARBOZA, J. S. Caracterização de Compósitos Magnéticos Macios Desenvolvidos Através da Matelurgia do Pó Aplicados a Núcleos de Máquinas Elétricas. Dissertação. PPGE3M: UFRGS, 2009.

[7] CREMONEZI, A.; KLEIN, A. N.; LOBERTO, A.; et al. A Metalurgia do Pó - Alternativa Econômica com Menor Impacto Ambiental. Grupo Setorial de Metalugia do Pó. Metallum Eventos Técnicos, 1a Ed., São Paulo, 2009.

[8] DIAS, M. M. Construção de Uma Máquina Síncrona de Relutância Trifásica com Rotor de Material Magnético Macio Sinterizado. Tese. PPGE3M: UFRGS, 1999.

[9] KRAUS, J.D. \& CARVER, K.R. Eletromagnetismo. R.Janeiro, Guanabara, 1978 $780 \mathrm{p}$

[10] NASAR, S.A. Handbook of Electric Machines. New York, McGraw-Hill, 1987.

[11] FITZGERALD, A.E, KINGSLEY, Jr. C, UMANS, S.D. Electric Machinery. New York, Bookman, 2008 (reimpressão), $6^{a}$ Edição.

[12] CATÁLOGOS SIEMENS. Motores de Corrente Continua - Guia rápido para uma especificação precisa, 2006, 36 p.

[13] STOREY, J. How Real Electric motor Work, University of New South Wales, Sydney - Austrália, 2009, 40 p.

[14]http://pessoal.utfpr.edu.br/mariano/arquivos/Transformadores_3.pdf, acessado em 12/06/2014.

[15] JILES, D.C. Recent advances and future directions in magnetic.materials. Acta Materialia, v. 51, p.5907-5939, 2003. 